Revista Arbitrada Interdisciplinaria KOINONIA

Año VI. Vol VI. N³. Edición Especial: Educación II. 2021

Hecho el depósito de Ley: FA2016000010 ISSN: 2542-3088

FUNDACIÓN KOINONIA (F.K). Santa Ana de Coro. Venezuela.

Wendy Elizabeth Rivadeneira-Ochoa; Luis Bolívar Cabrera-Berrezueta

http://dx.doi.org/10.35381/r.k.v6i3.1321

\title{
Rol docente y aprendizajes significativos
}

\section{Teaching role and significant learning}

\author{
Wendy Elizabeth Rivadeneira-Ochoa \\ wendy.rivadeneira.21@est.ucacue.edu.ec \\ Universidad Católica de Cuenca, Azogues \\ Ecuador \\ https://orcid.org/0000-0002-9264-6452 \\ Luis Bolívar Cabrera-Berrezueta \\ bolivarcabrera@ucacue.edu.ec \\ Universidad Católica de Cuenca, Cuenca \\ Ecuador \\ https://orcid.org/0000-0002-6853-635X
}

Recepción: 10 de abril 2021 Revisado: 05 de mayo 2021

Aprobación: 30 de junio 2021

Publicación: 15 de julio 2021 


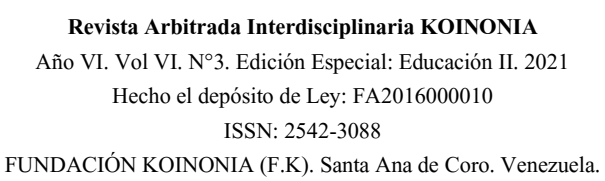

Wendy Elizabeth Rivadeneira-Ochoa; Luis Bolívar Cabrera-Berrezueta

\title{
RESUMEN
}

La formación profesional de un docente debe ser continua e indispensable para hacer frente a los nuevos retos y desafíos que lograrán mejoras innumerables en el aprendizaje del estudiante, la presente investigación tuvo como objetivo determinar el rol docente para mejorar el proceso de enseñanza-aprendizaje. Metodológicamente se desarrolló desde un enfoque cualitativo. Los resultados obtenidos destacan que los docentes aplican principalmente tres metodologías activas: Aprendizaje basado en problemas, proyectos y Aula invertida, por ello se diseñó la técnica del AMOR con el propósito de que sea empleada para un mejor aprovechamiento de estas metodologías que propiciarán en el estudiante el desarrollo de competencias y habilidades encaminadas a adquirir aprendizajes significativos y prepararlo para desenvolverse en el mundo que lo rodea.

Descriptores: Método de enseñanza; estrategias educativas; innovación pedagógica; aprendizaje activo. (Palabras tomadas del Tesauro UNESCO).

\begin{abstract}
The professional training of a teacher must be continuous and essential to face new challenges and challenges that will achieve innumerable improvements in student learning, the present research aimed to determine the teacher role to improve the teaching-learning process. Methodologically, it was developed from a qualitative approach. The results obtained highlight that teachers apply mainly three active methodologies: Learning based on problems, projects and Inverted Classroom, for this reason the LOVE technique was designed with the purpose of being used for a better use of these methodologies that will promote the student the development of competencies and abilities aimed at acquiring meaningful learning and preparing it to function in the world that surrounds it.
\end{abstract}

Descriptors: Teaching methods; educational strategies; teaching method innovations; activity learning. (Words taken from the UNESCO Thesaurus). 


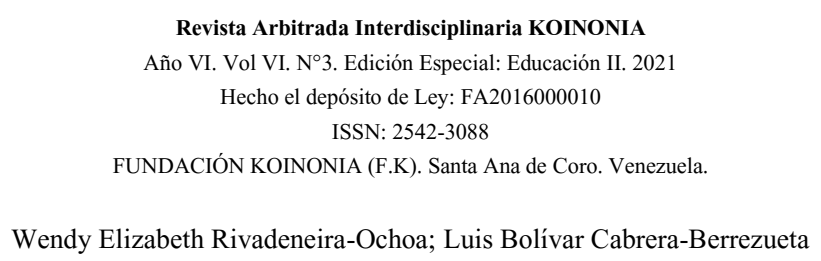

\section{INTRODUCCIÓN}

Empecé mi carrera dictando cursos a través del SECAP en la Provincia de Morona Santiago más o menos en el año 2010, trabajé como instructora en el área de Contabilidad, Tributación, Atención al cliente, Emprendimiento de pequeños negocios entre otros relacionados al área contable, en su mayoría dictaba estos cursos en áreas rurales de algunos cantones de mi provincia, pude notar que los estudiantes carecían de conocimientos básicos, requisito indispensable para tomar los cursos, lo que me impedía avanzar como estaba estructurado el contenido para nivelarlos en el curso a seguir, a pesar de ello, muchos de mis alumnos no captaban la materia, desde entonces siempre me pregunté si debía cambiar la metodología y pedagogía con la cual estaba trabajando. Hoy me puedo dar cuenta, que ser docente no es fácil, se requiere de constancia, de paciencia, de preparación pedagógica talvez yo no supe cómo llegar a mis alumnos porque en la universidad aprendí a ser contadora más no docente, sin embargo, continué dictando cursos y poco a poco fui mejorando mi pedagogía. Es por ello que escogí realizar éste, artículo porque quizá como yo, muchos docentes tenemos esa incertidumbre ¿seré yo quien debe mejorar?

En la escuela tradicional el profesor tenía un papel autoritario y transmisor de saberes, él era el único conocedor de la materia, el alumno era oyente de sus conocimientos y mientras mejor memorizaba el contenido mayor era su conocimiento, siendo esta la clave de su evaluación, su actuación era totalmente pasiva; sin duda, estas características de personalidad siguen existiendo no solo en alumnos sino también en docentes, ocasionando una educación decadente en pleno siglo XXI. (Rodríguez, 2013).

De acuerdo al (Consejo Económico y Social ECOSOC, 2011), será muy difícil alcanzar los objetivos de la EPT dando uso a mecanismos tradicionales de educación particularmente en países en vías de desarrollo. Por su parte la (UNESCO, 2013), afirma que el uso de las TIC generaría grandes impactos por las oportunidades de aprendizajes que a través de su uso se logra desde cualquier parte del mundo, concluyendo que 
mejorarían el proceso enseñanza-aprendizaje, impulsando el desarrollo de habilidades y competencias que la sociedad actual necesita.

La educación actual reclama cambios en el sistema, introducir nuevas y mejores formas de enseñar y aprender con el uso de herramientas, métodos y recursos que debido al veloz avance de la tecnología en todos sus espacios, aún más en el campo educativo exige que los docentes usemos las TIC como medio para lograr en el estudiante aprendizajes significativos a través de la creación de ambientes motivadores y mejorados en el que el estudiante desarrolle su alto potencial.

De acuerdo con (Martínez, 2018), no es solo el saber trabajar con herramientas informáticas, se debe impulsar habilidades y competencias en los estudiantes, brindándole mejores oportunidades que les permitan desarrollar a través de las TIC esas habilidades; utilizándolas como un camino con numerosas estrategias. Para lograr este cambio significativo en la educación el rol docente debe también cambiar, aprender a ser flexibles y estar abiertos a los constantes avances de la tecnología, considerando a las TIC una valiosa estrategia y oportunidad para mejorar su metodología y pedagogía durante el proceso enseñanza-aprendizaje, propiciando espacios que logren nuevas experiencias y aprendizajes significativos en los alumnos.

El Ministro de Educación y Deportes de la República de Argentina Esteban Bullrich alegó que la educación es la clave de avance y la libertad como única forma para dar poder a las personas, endurecer a las entidades y sostener a todas las personas, indicó también que el eje fundamental para educar con calidad es el docente, quién debe optar con innovadoras formas de enseñar (UNESCO-OREALC, 2017).

A través del presente artículo quiero llegar a todos los docentes interesados en construir una educación diferente, creando un camino mejor y alternativo de aprendizaje, dejar de lado el modelo tradicional y generar nuevos escenarios a través de los OVA con el uso de herramientas que aproxime a sus alumnos al manejo oportuno de la tecnología y el desarrollo de la creatividad. 


\section{Referencial teórico}

En este sentido, (Rojas-Moreno, 2016), menciona que: el uso de las TIC en el proceso educativo ayuda a que el estudiante pueda adquirir competencias y habilidades siendo el docente quien desempeña un papel importantísimo dentro de este proceso, al ser el mediador para que el educando logre alcanzar esas competencias y nuevos estilos de aprendizaje, proporcionando el ambiente adecuado que faciliten el uso de estas herramientas tecnológicas, ésta es la responsabilidad del docente, prepararse para brindar mejores oportunidades a sus alumnos con el uso de las TIC.

La realidad de nuestro país y del mundo es que para todo escenario la tecnología ha tomado un papel protagónico en la sociedad, tanto se ha vuelto dependiente de nosotros que según estadísticas en el año 2018 cada 60 segundos se vinieron produciendo 3,7 millones de búsquedas en Google, se enviaron 38 millones de WhatsApp, 18 millones de mensajes de texto, 481.000 tweets y 187 millones de email (García-Contador \& GutiérrezEsteban, 2020).

Estamos tan inmersos en la era digital que todo se mueve a través de ella, hoy en día los negocios giran por la red con el solo uso de una tarjeta y desde un celular, si hacemos un apartado al pasado como nos comunicábamos, como se escuchaba música, como se veía noticias, películas, como se transportaba la gente, las clases eran únicamente presenciales, los niños jugaban con otros en las calles, en fin a todos estos cambios a los que nos hemos enfrentado se los Ilama disrupción (García-Contador \& GutiérrezEsteban, 2020).

En los nuevos modelos de educación las herramientas tecnológicas tienen un papel primordial sobre todo en lo informático que desde 1970 han generado grandes cambios, por ello algunos autores mencionan innovaciones pedagógicas disruptivas ocasionadas por el uso de las TIC en el campo educativo (Acevedo, 2015). Todo proceso de enseñanza y aprendizaje que integra las TIC tiene ciertas características: está dirigido a todos los estudiantes; forma habilidades, destrezas, actitudes y valores; se deriva de la realidad y se integra en todas las disciplinas estudiadas en las escuelas; valora los 
recursos del tiempo asignado a la educación artística y las habilidades interculturales; hace que el aprendizaje sea más agradable y más relevante para la vida cotidiana de los educandos y ofrece la oportunidad de compartir información dentro de un grupo; desarrolla habilidades de comunicación con las demás personas y fomenta el respeto y la cooperación en proyectos conjuntos y apoya la cohesión social; garantiza igualdad de oportunidades de participación para todos los estudiantes involucrados en el proceso educativo (Munteanu et al. 2014).

Es necesario reflexionar en una propuesta con inclusión de las TIC en el proceso enseñanza aprendizaje, no como una herramienta nada más, sino como un elemento indispensable para el inicio de una educación disruptiva que nos permita alcanzar un cambio total en nuestras prácticas educativas, generando un sistema diferente en el cual el objetivo principal sea el de ofrecer una educación de calidad para que el educando desarrolle aprendizajes significativos.

En este sentido, (Serin, 2017), uno de los elementos más importantes en el desarrollo de la pasión por la docencia es el compromiso y dedicación de los profesores a los estudiantes y su aprendizaje. Los maestros apasionados están fuertemente comprometidos con su trabajo y pueden inspirar y despertar en sus estudiantes el deseo de aprender.

Con la aplicación de metodologías activas el docente deja de ser la fuente de conocimiento convirtiendo al educando en el centro del aprendizaje como protagonista, afirma que el educador pasa a orientar, organizar, aportar ideas y criterios, que los estudiantes a través de la aplicación de metodologías se involucran para aprender constantemente y de manera colaborativa desarrollar en ellos valores de respeto y tolerancia, convirtiéndoles en personas creativas capaces para desarrollar su pensamiento crítico y reflexivo, potenciándolos para adaptarse al cambio; concluye diciendo que el sistema educativo debe facilitar el aprendizaje de cosas diferentes así también la enseñanza de manera distinta, con el propósito de satisfacer a los educandos que van evolucionando con la sociedad (Matzumura-Kasano et al. 2018). 
El Aprendizaje Basado en Problemas ( $\mathrm{BBP}$ ) viene a ser una táctica encaminada a contrarrestar los diversos problemas que conlleva la enseñanza del pasado (Colón \& Ortiz, 2020). Por lo tanto, es un método de aprendizaje que funciona con situaciones problemáticas, elaborado previamente por el equipo de profesores, que tiene como objetivo estimular procesos cognitivos en relación con un sujeto determinado. Problemas que son cuidadosamente planificados por un grupo colaborativo que tiene como objetivo integrar contenidos teóricos y se distribuyen en el plan de estudios matriz de acuerdo con una secuencia de aprendizaje, es decir, con el crecimiento de grados de complejidad (Silva-da-Rocha-Marques, 2018).

El Aprendizaje basado en proyectos es una estrategia muy utilizada en todos los niveles educativos, funciona frecuentemente con pequeños grupos que trabajan de manera colaborativa y todos son responsables de lograr el objetivo, el resultado es que los educandos desarrollan niveles más hondos de comprensión y nuevas habilidades, esta metodología de aprendizaje demanda que sus alumnos sean activos y estén comprometidos en la construcción de su propio aprendizaje (Toledo \& Sánchez, 2018). En este sentido "el Aula invertida es una metodología que consiste en invertir las técnicas de aprendizaje que se daban en el aula, para trasladarlos a su exterior, lo que quiere decir trabajarlos en la casa, y a la inversa" (Gonzalez et al. 2020), por consiguiente, la nueva educación debe generar un cambio importante en el proceso enseñanzaaprendizaje, en el cual el educador y su participación activa es la pieza fundamental en el uso de nuevas metodologías.

De los resultados obtenidos de la investigación realizada por (Cedeño, 2019), se obtuvo que: la calidad de la educación no depende de manera directa del uso de las TIC empleadas en el proceso, sino de la metodología bajo la cual se involucran el uso de las TIC como nuevas herramientas de aprendizaje y también las actividades de aprendizaje elaboradas, se obtuvo que incluir elementos tecnológicos en el proceso de enseñanza contribuye a que se generen aprendizajes significativos en todas las áreas, debido a que facilitan al alumno un aprendizaje basado en la experiencia y el pensamiento crítico 
considerando que el aula no es el único espacio de aprendizaje y que el estudiante asimila nuevos conocimientos en entornos innovadores en el cual él es quien construye su propio aprendizaje.

David Ausubel es el creador de la teoría del aprendizaje significativo, según (Rivera, 2004), el aprendizaje significativo se da cuando las personas se relacionan entre sí intentando dar sentido al mundo que los rodea, éste aprendizaje construye las representaciones personales significativas y que poseen sentido de un objeto, situación o representación de la realidad, cuando lo que se trata de aprender se logra relacionar de forma sustantiva y no arbitraria con lo que ya conoce quien aprende, es decir, con aspectos relevantes y preexistentes de su estructura cognitiva.

\section{Capacitación Docente}

La formación docente dejó de ser importante en la escuela tradicional lo que ocasionó que el educador no sea el apoyo que el educando necesita para que desarrolle sus competencias y habilidades (UNESCO, 2010). Uno de los grandes retos que hoy en día tiene el docente es el aprender a usar la tecnología dentro del ámbito educativo, el docente tradicional aprendió y se formó para ser un docente de aula, en un espacio cerrado.

Hoy, el avance de la tecnología cambia este paradigma y nos obliga a todos los educadores a dejar de resistirnos a estos cambios bruscos por así decirlo para mejorar el proceso enseñanza aprendizaje, el buscar capacitación fortalecerá nuestros conocimientos como docentes, debemos ser realistas y admitir que los tiempos han cambiado que la sociedad requiere cambios. Ahora para la mejora del proceso enseñanza aprendizaje, el docente que no usa tecnología es un docente tradicional que no aporta al crecimiento de la sociedad, pues es bien sabido que la educación es el desarrollo de la misma.

Considero importante detenernos y hacer un análisis acerca de la manera en como el docente enseña con el uso de la tecnología, el gestionar la superación y la motivación 
del docente para incorporar en el proceso el uso de las TIC es innovador, por ello que (García-Sánchez et al. 2017), insisten en que es indiscutible la necesidad de buscar el perfeccionamiento en la capacitación de las TIC. Por otro lado, (Cabrol \& Severin, 2015), mencionan que: para el BID, inversión en TIC es el esfuerzo para que los gobiernos ofrezcan una educación de calidad a los educandos, que mejoraría el proceso enseñanza aprendizaje empezando por los contenidos del currículo y el desarrollo de habilidades y competencias que son necesarias para la sociedad del conocimiento.

Así mismo, la (UNESCO, 2013) indica que en una primera instancia actualizarse es un reto pedagógico, que lleva a incluir TIC en el salón de clase, en el currículo escolar, la adecuación de la formación inicial y en servicio de los docentes, políticas públicas seguras que implementen sistemas de reformas que logren cambiar el sistema educativo completamente, junto con esto el dotar de infraestructura tecnología en los establecimientos educativos propician el uso de las TIC para mejorar también la gestión escolar que viene de la mano con la capacitación de directivos y administrativos.

Nuevas pedagogías con inclusión de las TIC, logrará que las clases sean interactivas, estimulará el trabajo colaborativo y grupal logradas de las competencias adquiridas por el docente e indudablemente mejorará el proceso de aprendizaje de sus educandos (UNESCO, 2010). La formación profesional del docente será componente fundamental de esta mejora de la educación. No obstante, el desarrollo profesional del docente sólo tendrá impacto si se centra en cambios específicos del comportamiento de este en la clase y, en particular, si ese desarrollo es permanente y se armoniza con otros cambios en el sistema educativo (UNESCO, 2017).

\section{El quehacer del docente}

El docente debe tener capacidad de innovar, es decir inventar o crear algo diferente y mejor de lo que ya hay, tomando como base lo existente para mejorarlo centrarse específicamente en como el docente debe aplicar esta innovación en el ámbito educativo, teniendo a nuestro favor el uso de nuevas tecnologías de la información y la comunicación 
(TIC). La Innovación didáctica tiene relación con la concreción tanto en lo que refiere a planificar y cumplir las políticas educativas, asociada a los modelos para relacionarnos, en la infraestructura como aquella que sostiene mejora en la realización de actividades educativas y del proceso enseñanza-aprendizaje, se entiende como todos los cambios necesarios y que se introducen en el proceso, asociación a reformar el contenido curricular, metodología, medios, formas, y procesos o técnicas de evaluar ocasionando interacción en la relación docente-alumno para aproximarlos a los requerimientos del modelo pedagógico educativo y las necesidades de la sociedad.

La innovación tecnológica es el enseñar sistemas dentro de los entornos en todos sus niveles, establecer nuevas tecnologías en todas las asignaturas como espacios virtuales y salas de conferencias web, chats, foros, Padlets, entre otras como aquella que conlleva procesos de profundización e innovación de recursos y herramientas, también con el propósito de una mejora en el proceso enseñanza-aprendizaje (Zayas-Barreras et al. 2015).

Innovaciones que juntas están encaminadas a la generación de cambios significativos en el aprendizaje, a través de nuevos roles en los docentes para crear materiales, innovar métodos, cambiar contenidos o contextos en mejorar la calidad de enseñanza hacia el educando, lo que se busca es orientar al docente a innovar nuevas formas de enseñar, aprender y evaluar, aplicar correctamente metodologías activas como Aula invertida, Análisis de casos, Aprendizaje colaborativo, Aprendizaje basado en proyectos, Juego de roles, entre otras que ayudan a mejorar los resultados conjuntamente con el uso de nuevas herramientas y recursos en todas sus escenarios.

Esta es la era en la que ejercer la docencia requiere de preparación para entregar a sus educandos nuevas oportunidades para aprender con la ayuda de las TIC, el saber cómo utilizarlas y conocer acerca de su aporte en el aprendizaje de los alumnos es una de las competencias indispensables en el docente, para que a su vez centremos al estudiante en el importante uso de las TIC por las ventajas innumerables al aplicarlas. La enseñanza 
requiere de docentes que tengan competencias y dominen el uso de las TIC para que se logre enseñar con eficiencia las diferentes asignaturas (UNESCO, 2010).

No se puede hablar de nuevas tecnologías sino se aplica nuevas didácticas que le dan más valor a la competencia profesional de un docente de élite, que entusiasme, despierte interés y curiosidad, que estimule la creatividad y la construcción del conocimiento rompiendo paradigmas y perjuicios pasados, didácticas que inciten autonomía en el estudiante, interacción y participación en el aula, respondan a sus intereses, expectativas y necesidades, no únicamente del alumno sino también del docente, hoy el docente entra en una etapa importante de transición de enseñanza educativa.

\section{Compromiso de ser docente}

Una educación con valores es el aprendizaje más relevante que un docente debe demostrar y transmitir dentro y fuera del entorno de clases y que en la actualidad se ha dejado de lado por completo en estas nuevas generaciones que son quienes se desenvolverán profesionalmente en la sociedad desde ahora.

\section{Responsabilidad del docente}

Después de haber realizado una profunda investigación puedo describir las responsabilidades que un docente debe desempeñar, fijando su labor en cumplirla con amor y paciencia, entre ellas las siguientes: 


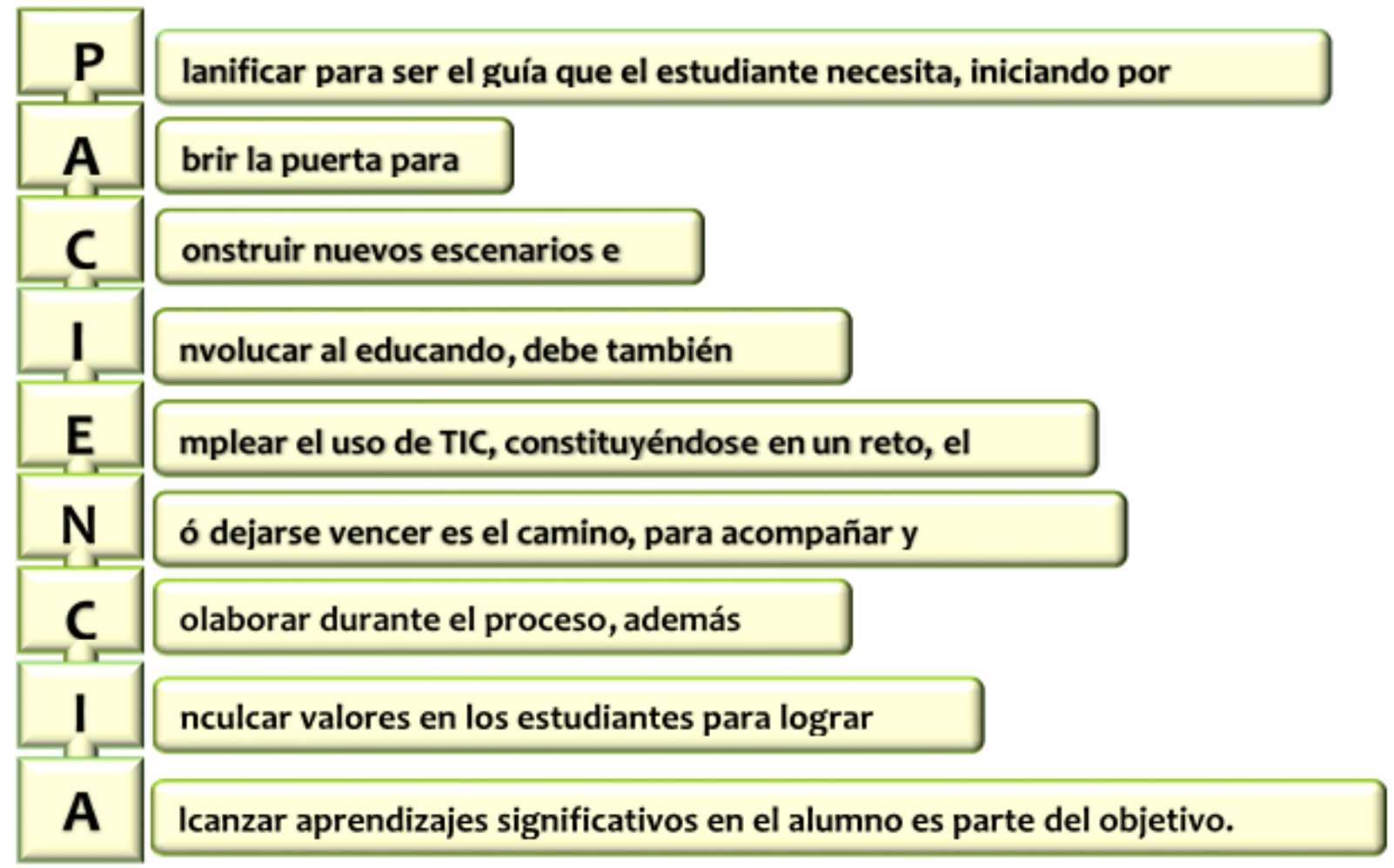

Figura 1. Responsabilidades del docente Fuente: Elaboración propia.

\section{METODOLOGÍA}

Desde un enfoque cualitativo se realizaron entrevistas a docentes pertenecientes al nivel de educación, Elemental, Preparatoria, Básica, Media, Superior y Bachillerato; para posteriormente construir las categorías que permitieron el análisis del fenómeno abordado. 


\section{RESULTADOS}

Se presentan los hallazgos de la investigación:

Tabla 1.

Análisis de resultados cualitativos.

\begin{tabular}{|c|c|c|}
\hline $\begin{array}{l}\text { Unidad de } \\
\text { Análisis }\end{array}$ & Categorías & Segmento \\
\hline $\begin{array}{l}\text { Herramientas } \\
\text { Tecnológicas y } \\
\text { recursos. }\end{array}$ & $\begin{array}{l}\text { Nombres de } \\
\text { herramientas } \\
\text { tecnológicas y } \\
\text { recursos que } \\
\text { utiliza. } \\
\text { Uso de } \\
\text { herramientas } \\
\text { tecnológicas y } \\
\text { recursos. }\end{array}$ & $\begin{array}{l}\text { Alto, porque me encuentro en el área urbana. } \\
\text { No tengo acceso por cuanto al ser un } \\
\text { establecimiento rural no hay internet. } \\
\text { He escuchado de ellas, pero en el área que } \\
\text { me encuentro trabajando no es útil usarlas } \\
\text { porque no se tiene accesibilidad a ellas, los } \\
\text { PPFF no tienen teléfonos inteligentes son de } \\
\text { escasos recursos económicos. } \\
\text { No hay herramientas tecnológicas en los } \\
\text { establecimientos educativos para lograr } \\
\text { calidad de educación pero como docente me } \\
\text { adapto a lo que hay. } \\
\text { Internet, Computadora, Celular. } \\
\text { Plataformas: Esemtia, Edebeon, Teams, } \\
\text { Zoom, Proyector, Prezi, Redes sociales, } \\
\text { Wasap, Correo electrónico, Messenger, } \\
\text { YouTube, Genially, Padlet, PowerPoint, } \\
\text { Infografías, Base 10, Láminas } \\
\text { Siempre, en todo momento, una combinación } \\
\text { entre herramientas tecnológicas y recursos. } \\
\text { Actualmente se usa mucho en la planificación } \\
\text { y el desarrollo de la clase. } \\
\text { No se usa por cuanto no hay internet. } \\
\text { Hay muchísimas herramientas y recursos } \\
\text { tecnológicos que enriquecerían nuestra labor, } \\
\text { pero se debería contar con internet de calidad }\end{array}$ \\
\hline
\end{tabular}


Metodologías Activas
Conocimiento de metodologías activas.

Metodologías que aplica.

Conocimiento del entorno del estudiante. para que sean aprovechadas de manera óptima.

Importancia del Poco frecuente por la materia que imparto.

uso de

Herramientas

tecnológicas y

recursos.

Muy necesarios para que el estudiante se apodere del conocimiento y construya su propio aprendizaje.

Muy adecuadas para sorprender al estudiante con una clase interactiva, y de calidad.

Gracias a las herramientas el docente tiene la información a la mano, dispone de recursos novedosos pero hace falta la capacitación.

Únicamente conozco las básicas, no he revisado acerca de otras metodologías activas.

Poco, no me he visto en la necesidad de buscar información acerca de metodologías activas.

Si conozco, pero por mi materia no he usado variedad de metodologías activas.

Aprendizaje basado en problemas, Aprendizaje basado en Proyectos, Aprendizaje Colaborativo.

Aula invertida, Trabajo autónomo, Estudio de Casos, Métodos de observación: concentración, aplicación de procesos inductivos, demostrativos, de aprendizaje rápido, de captación a través de cuadros sinópticos elaboración de experiencias vividas, criterios teóricos, diálogo, debate, refuerzo para luego evaluar.

Si conozco, no en su totalidad, pero lo necesario. 
Importancia de conocimiento del entorno del estudiante.

Rol del docente enseñanza aprendizaje
Si debido a que cada estudiante cuenta con una ficha de información en cuanto a dirección, situación económica, servicios básicos, entre otros.

Es complejo conocer el entorno de todos los estudiantes por el alto número con el que se trabaja.

No por cuanto no soy tutor. La comunidad es pequeña por ello si se conoce en su totalidad.

Primero hay que ser humanos y luego profesionales es primordial conocer el entorno del estudiante, su estado social y emocional, para comprender y entender su realidad.

Como docentes tenemos la obligación de percibir actitudes en el estudiante y apersonarnos de ellas para saber cómo llegar a él.

Muy importante para saber cómo impartir las clases, que materiales solicitar y cómo trabajar con ellos.

Orienta, guía, acompaña, aclara las dudas, facilita el aprendizaje del estudiante, El estudiante es nuestra razón de ser, él es nuestro rol.

Ser una figura cercana al estudiante, generar un ambiente de confianza.

Prepararnos, capacitarnos, evolucionar la forma de educar, optar por nuevas estrategias y métodos.

Dominar las tecnologías.

Afrontar retos y desafíos de la profesión. Formar mejores personas y grandes profesionales. Motivar al estudiante. 
Evaluación del estudiante

Informes y

Planificación del docente
Aspiraciones en cuanto al currículo y sistema.
Cumplir con el papel de ser un segundo padre para el alumno.

Enseñar no solo el conocimiento sino también en valores y principios.

Mediante la aplicación de pruebas.

A través de su comprensión durante el desarrollo de la clase. Fichas de observación con trabajo en clase.

Listas de cotejo. Rubricas de evaluación.

Instrumentos de evaluación: pruebas sumativas, de diagnóstico, orales, de selección múltiple.

A través de Google Fomrs.

Los informes, planificaciones se deben cumplir y ajustar a las exigencias del distrito porque es parte de nuestra labor.

Las elaboraciones de tantas planificaciones están de más, es agobiante, estresante y abrumador debería reducirse el papeleo

Los informes son exigencias que deberían reducirse para ese espacio de tiempo utilizarlo en preparar mejor una clase activa, motivada, de calidad.

Se sobresatura al docente con actividades que no debería realizar: llenado de matrices, presentación de información, evidencias de actividades realizadas, se debería designar otro profesional para las actividades administrativas, el docente debe centrarse en su desempeño como tal.

Qué el currículo sea flexible, la educación debe ser gratuita para todos, el sistema debe preocuparse por atender a todos los niños que están en la calle, por tener estudiantes 
motivados, padres de familia con trabajo para que haya una mejor alimentación en sus hogares.

Los textos deben apegarse a la realidad de cada lugar.

Eliminar exámenes remediales y de gracia estas oportunidades hacen que el estudiante pierda el interés por educarse.

El estado debería invertir más en educación y asignar más presupuesto para contribuir en la mejora del proceso enseñanza aprendizaje, dotando de infraestructura en los establecimientos educativos, espacios físicos, recursos innovadores, entre otros.

Se debe mejorar la remuneración del docente, tenerlo tranquilo, contento y descansado, merecemos salir de vacaciones sin tanta presión,

Apoyar al docente para su desarrollo y formación profesional.

El sistema debería enfocarse a desarrollar la ética, fomentar la lectura, enseñar valores, principios para ello darse un cambio estructural de la visión de quienes están al frente de la educación.

Reanudar los bachilleratos técnicos, que hacen que el estudiante aprenda de manera significativa.

El estado debe proveer especialistas para atender las NEE en cada establecimiento. Reactivar la escuela para padres que son de

Fuente: Entrevistas. gran ayuda en educación. 
Con base a los criterios remitidos en la unidad de análisis herramientas tecnológicas y recursos categoría acceso a herramientas tecnológicas y recursos, se puede evidenciar que son pocos los docentes que tienen acceso a ellas debido a diversas limitaciones, falla de internet, poca infraestructura en los establecimientos educativos, entornos rurales, costos, desconocimiento de su aplicación entre otros aspectos optando finalmente por trabajar con lo que tienen.

Dentro de la misma unidad de análisis en cuanto a la categoría nombres y uso de herramientas tecnológicas y recursos, los docentes están aplicando herramientas básicas y otras que la institución dispone debido a que se ven limitados a ellas por sus costos, por la falta de una oportuna capacitación y por cuanto trabajan con aplicaciones que sean de fácil acceso al estudiante, finalmente el objetivo es llegar a él sin considerar las limitaciones que en el párrafo anterior mencionábamos; en lo que pueden, hacen una combinación entre herramientas tecnológicas y recursos que se disponen en el entorno. En la categoría Importancia del uso de Herramientas tecnológicas y recursos, todos los docentes concuerdan en apreciar que las mismas provocan en el proceso una educación activa y mejorada y convienen en decir que son un mecanismo enriquecedor y activo que provoca ambientes más interactivos para el estudiante cautivando su atención hacia la construcción del conocimiento.

Al encontrarnos en una sociedad cada vez más tecnológica nuestra labor docente debe ir a la par, debemos ir mejorando nuestro conocimiento, perfeccionando la aplicación de estas herramientas y recursos que hoy en día se ofrecen en el campo educativo innovación, capacitarnos para estar preparados y que no sorprendan nuestro ego sino más bien las percibamos como un apoyo en el proceso enseñanza aprendizaje sin miedos sin restricciones, pero sobre todo sin limitaciones.

(García-Sánchez et al. 2017), mencionan que no se trata solo de usar TIC sino de saber detectar cuándo, cómo, y para qué usarlas, lo que permitirá al docente mejorar su habilidad y ahondar en su conocimiento a través de estas herramientas científicas, manifiesta que esto se logra siempre que el docente este en continua formación y 
dispuesto a dirigirse al perfeccionamiento de manera individual y grupal para provocar el desarrollo de sus motivaciones, habilidades, capacidades y competencias.

En base a los criterios emitidos en la unidad de análisis metodologías activas categoría conocimiento de metodologías activas y metodologías que aplica, hay diferentes puntos de vistas puesto que los docentes conocen poco de ellas, no ven la necesidad de aplicarlas, otros conocen pero no existe la necesidad de variar su uso por ello aplican las más conocidas, otros aplican la experiencia sin conocer qué tipo de metodología usan, y otros tienden a una confusión entre metodologías activas y recursos, sin embargo a través de mi investigación puedo observar que en su mayoría los docentes coinciden en aplicar Aprendizaje Basado en Problemas, Aprendizaje Basado en Proyectos y la conocida Aula Invertida.

Nuestra labor como docentes debe estar dirigida al estudiante poniendo a su alcance herramientas, contenidos, estrategias, metodologías y recursos necesarios para desarrollar su mayor potencial y puedan desenvolverse como buenas personas y excelentes profesionales en el medio que los rodea, debemos educar encaminando al estudiante para que esté listo de abrirse al mundo real y enfrentar diferentes problemas y situaciones.

En cuanto a la unidad de análisis entorno del estudiante y sus categorías conocimiento del entorno del estudiante y su importancia, pocos no conocen el entorno del estudiante, pero la mayor parte de docentes entrevistados indican que sí y que esto es fundamental y estratégico para el proceso enseñanza aprendizaje.

Los docentes por compromiso propio debemos saber ponernos en los "zapatos del otro", concientizarnos de que cada alumno vive un mundo diferente, economía baja, media, alta, con diferentes culturas y costumbres, atender las necesidades de nuestros educandos es responsabilidad del estado pero comprender su estado emocional, conocer su nivel económico, saber si desayunó, padres divorciados, padres migrantes, en fin conocer su entorno, es desde mi punto de vista estrictamente necesario del docente, debido a que todos estos factores del entorno afectan la construcción del conocimiento 
en el educando, y debemos actuar ante ello para generar un ambiente de confianza en el que el estudiante sienta que es importante su vida así como también su educación.

Para culminar el desarrollo de mi investigación he visto necesario enfatizar en la unidad de análisis proceso enseñanza aprendizaje y, en su categoría rol del docente, los entrevistados armonizaron en que el rol ha cambiado y que deben estar preparados para afrontar retos y desafíos y así cumplir con el papel de orientador, guía, facilitador, poniendo al educando como el protagonista de su propio conocimiento construyendo así, un alumno listo para desarrollarse en la vida no sólo académicamente sino también formando mejores personas, pero manifiestan que no son apoyados por el gobierno para recibir de manera oportuna capacitaciones que los ayuden en su formación.

En la categoría evaluación del estudiante, los docentes evalúan el conocimiento; en su mayoría es a través de pruebas con el uso de instrumentos como: rubricas de evaluación, listas de cotejo, fichas de observación, participación en clase, trabajos en clase, y una minoría utiliza el google forms como herramienta de evaluación.

A través de la categoría Informes y Planificación del docente, manifiestan su descontento debido a que la carga es demasiada y esto provoca situaciones emocionales e incluso no saludables en el desempeño de su rol, dicen estar conscientes en que es necesaria una planificación y uno que otro informe pero que la excesiva presentación de ello obstaculiza dedicar el tiempo necesario en planificar una clase innovadora, consideran que esto es un trabajo de otro profesional dentro del ámbito educativo.

Por último he creído muy importante en el desarrollo de mi artículo resaltar como categoría aspiraciones en cuanto al currículum y el sistema en la cual los docentes concordaron en manifestar: las personas que están al frente de la educación deberían realizar un cambio estructural de su visión, atender al campo educativo con acceso a una educación para todos y que ésta sea gratuita, asignar más presupuesto en las zonales y que su distribución sea equitativa, eliminar los exámenes remediales y de gracia, que los textos se acoplen a la realidad de cada provincia, dotar a todos los 
establecimientos educativos con expertos en NEE, con infraestructura y tecnología, mejora salarial, formación profesional continua del docente, y enfocarse en fomentar una educación con valores, fomentar la lectura, apertura el bachillerato técnico.

Como docentes debemos pensar en un nuevo prototipo de educación en el cual dejemos de lado la teoría para poner en práctica saberes y experiencias que es lo que romperá con la educación tradicional que sigue siendo parte de nuestra pedagogía, un modelo que nos permita utilizar mejores recursos, aplicar diferentes metodologías y descubrir herramientas constructivas para el aprendizaje del alumno.

El docente es la persona que educa, guía, enseña, impulsa a niños y jóvenes, que a diferencia del docente pasado hoy en día el docente tiene un valor agregado, a este valor agregado es a lo que yo lo llamo TIC. El rol que debe tener un docente requiere de compromiso y amor por enseñar a los demás, el docente es el pilar más importante en la educación y como tal el rol que debe cumplir lo es aún más, porque de él depende la formación personal, académica y profesional del educando, me encanta esa frase que dice "Nadie olvida a un buen profesor" eh ahí la dedicación, esmero y vocación del ser docente.

Indiscutiblemente es un sentir común que no se agobie tanto al docente con exigir la presentación excesiva del papeleo y que a cambio se dedique más inversión en educación, en este último aspecto pienso que no solo los docentes aspiramos eso, también estudiantes, padres de familia y todo un país necesitado de prosperar, cansado de limitar las cosas verdaderamente importantes para el crecimiento de la sociedad.

\section{PROPUESTA}

A través de los resultados obtenidos en el presente estudio puedo evidenciar que los docentes necesitan de programas de capacitación que los ayude a formarse profesionalmente no solo en cuanto a TIC, también en la aplicación de metodologías activas para adoptar nuevos roles que les permitan adquirir habilidades y competencias que generan ambientes diferentes y renovados dentro y fuera del aula, del estudio 
realizado se obtuvo que los docentes aplican principalmente tres metodologías: Aprendizaje basado en problemas, Aprendizaje basado en proyectos y Aula invertida, por tal razón mi prepuesta va encaminada en mejorar el uso de estas metodologías plasmando mi artículo en que juntas sean aprovechadas potencialmente en el proceso enseñanza aprendizaje mediante la intervención guiada del docente con la implementación de la técnica del AMOR para generar "Aprendizajes Significativos" en el estudiante.

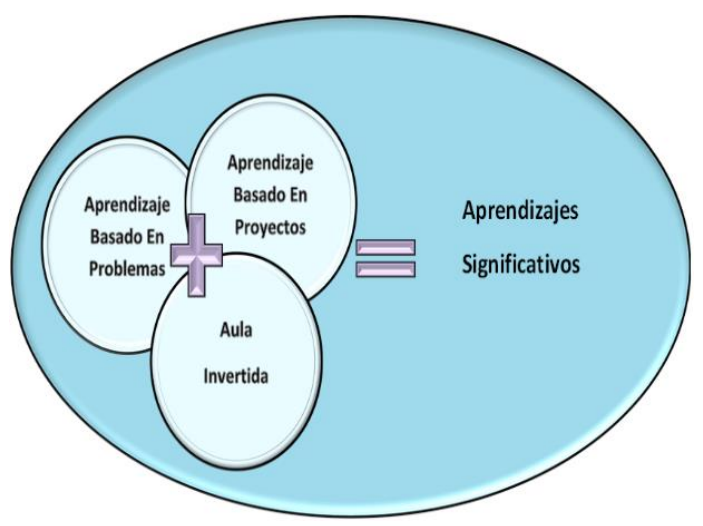

Figura 2. Rol docente para generar aprendizajes significativos. Elaboración: Los autores.

Las metodologías activas son estrategias que utilizan los docentes para la mejor compresión de un tema de cualquier asignatura, para dinamizar de manera participativa el desarrollo de un contenido, para sorprender al estudiante captando su atención etc., estas metodologías activas provocan en el estudiante descubrir nuevos conocimientos y construir su propio aprendizaje desde los diferentes escenarios, acciones, actividades, métodos y estrategias planteadas.

Incuestionablemente tres metodologías enriquecedoras para el proceso enseñanzaaprendizaje que profundizando en su aplicación me llevaron a gestionar un producto valioso para el docente, y a través de las oportunas guías recibidas lanzar ésta propuesta que persigue desarrollar competencias en el educando construyendo su propio conocimiento por medio de la creatividad y experiencia propia, valorando 
habilidades que deben ser respaldadas por el educador para generar en él aprendizajes significativos utilizando la técnica del AMOR, sus pasos son:

Abrir la puerta.- es decir motivar, desde mi percepción no es opcional es una ley del docente, cinco minutos que pueden despertar el interés, mejorar el estado emocional y generar un ambiente activo en los estudiantes previo al conocimiento, para ello es necesario realizar actividades si hay disponibilidad con recursos tecnológicos de lo contrario con los que en el medio existan, no es lo mismo decir jóvenes buenos días el día de hoy hablaremos de..... que empezar diciendo buenos días como están, nos espera un día interesante pero antes empezaremos con una dinámica, realizar una oración fomentando también principios que son importantes para su crecimiento como persona; o, también el dedicar un espacio al diálogo es alentador para conocer los intereses del alumno, y luego empezar con una lluvia de ideas e ir entrándome poco a poco en la materia.

Misión.- considero que es el paso más importante, el docente debe guiar y facilitar el aprendizaje mediante la asignación de funciones y roles que el estudiante debe considerar y con la búsqueda minuciosa de información que será seleccionada luego de haber realizado una investigación previa y la interacción de los involucrados en el proceso fortalecerá su conocimiento, como dice la frase "lo aprendido nunca se olvida", es decir éste es el camino que le permitirá al estudiante construir colaborativamente su aprendizaje. En esta etapa el docente entrega la rúbrica al estudiante y a través de una adecuada alfabetización informacional le proporcionará al estudiante estrategias de búsqueda y almacenamiento de valioso contenido que será debatido en el entorno para su aprendizaje significativo.

Organización. - la construcción del aprendizaje de contenidos y competencias debe estar claramente definido, para brindar a los educandos diversas oportunidades de colaboración y reflexión de las experiencias perfeccionadas con sus compañeros de clase, el docente debe involucrar al estudiante en lo relacionado aprender a ser, aprender 
a aprender, aprender a hacer y aprender a vivir en sociedad desarrollando contenidos de la cultura local, nacional, y mundial.

- Aprender a ser.- es lo que le da sentido al aprendizaje y accionar humano determinado por los esfuerzos académicos

- Aprender a aprender.-.aplica contenidos como: conceptos, datos, principios, esquemas es decir lo relacionado al conocimiento.

- Aprender a hacer.- se refiere a las capacidades, destrezas y habilidades.

- Aprender a vivir.- es el comportamiento que adoptan las personas frente a cualquier situación, aquí se resaltan los valores y actitudes aprendidas.

Retroalimentación.- es necesario que el docente realice este paso en todas las etapas anteriores acerca de los contenidos atendidos, si bien el rol del docente debe ser el guía deberá acompañarlo en todo el proceso para que sea el apoyo que el estudiante necesita en todo momento, es decir no se trata de dar una función y dejar que el solo se ahogue en el mar, el docente debe ser quien dirija el barco hasta que el alumno llegue a obtener el aprendizaje deseado esto ayuda al educando a crear diferentes ideas y caminos para aclarar sus dudas a la consecución del objetivo que es el lograr aprendizajes significativos.

\section{CONCLUSIONES}

El progreso de un país es la educación, el docente es un pilar muy importante en el proceso, una educación de calidad requiere la oportuna formación personal y profesional del educador quien deberá asumir nuevos roles que lo ayudarán a usar la tecnología con destreza, habilidad y confianza, mejorando su instrucción y pedagogía, creando nuevos espacios de aprendizaje con la ayuda de herramientas y recursos novedosos que hagan sus aulas más interesantes y propiciando que el alumno desarrolle habilidades y competencias para prepararlo a la consecución de metas y objetivos planteados por el para su vida. 
La investigación y proceso para la obtención de la información objeto de estudio, inicialmente fue planificada para realizarla a través de grupos focales, pero la situación que actualmente se vive por el COVID-19 dificultó el proceso debido a que los docentes están en constantes reuniones, las clases son impartidas en la mañana o en la tarde ocasionando que se realizaran las entrevistas de manera individual y extendiendo un poco más el cronograma establecido.

Los resultados obtenidos mediante el trabajo de investigación, fueron indicios para determinar que es necesario la capacitación y formación de los docentes en cuanto a herramientas tecnológicas, recursos y metodologías activas, el docente debe aprender a dominar la tecnología y con la aplicación de metodologías activas innovar y aprovechar los diversos escenarios que pueden propiciar en sus aulas.

El ser docente nace del corazón y la profesión de enseñar debe ser guiada con paciencia y amor por lo que se hace, he creído conveniente y necesario implementar una técnica que de realce al proceso con la aplicación de metodologías activas que generan aprendizajes significativos en los estudiantes, la meta que persigue el docente, es ser un mejor docente, la técnica del AMOR servirá de guía para conseguirlo, pero esto no tendría efecto si el docente pone obstáculos en cuanto a su formación profesional, que hemos visto es de vital importancia para su crecimiento.

\section{FINANCIAMIENTO}

No monetario.

\section{AGRADECIMIENTO}

A la Jefatura de Posgrados de la Universidad Católica de Cuenca por permitir el desarrollo y fomento de la investigación. 
Revista Arbitrada Interdisciplinaria KOINONIA

Año VI. Vol VI. N³. Edición Especial: Educación II. 2021

Hecho el depósito de Ley: FA2016000010 ISSN: 2542-3088

FUNDACIÓN KOINONIA (F.K). Santa Ana de Coro. Venezuela.

Wendy Elizabeth Rivadeneira-Ochoa; Luis Bolívar Cabrera-Berrezueta

\section{REFERENCIAS CONSULTADAS}

Acevedo, Á. (2015). Nuevas enseñanzas disruptivas en la educación superior en ciencias sociales. Los cursos en línea masivos y abiertos (MOOCs) [New Disruptive Teachings in Higher Education in Social Sciences. Massive and open online courses]. Revista Temas, 3(9), 125-136. https://doi.org/10.15332/rt.v3i9.1366

Cabrol, M., \& Severin, E. (2015). TICS en educación: una innovación disruptiva [TICS in education: a disruptive innovation]. https://n9.cl/io793

Cedeño, E. (2019). Entornos Virtuales de Aprendizaje y su rol innovador [Virtual Learning Environments and their innovative role]. ReHuSo: Revista de Ciencias Humanísticas y Sociales, 4(1), 119-127.

Colón, L., \& Ortiz, J. (2020). Efecto del Uso de la Estrategia de Enseñanza Aprendizaje Basado en Problemas (ABP) en el Desarrollo de las Destrezas de Comprensión y Análisis de la Estadística Descriptiva [Effect of Using the Problem-Based Learning (PBL) Teaching Strategy on the Develop. Revista Iberoamericana de Evaluación Educativa, 13(1), 205. https://doi.org/10.15366/riee2020.13.1.009

Consejo Económico y Social ECOSOC (2011). Educación de calidad en la era digital. Una oportunidad de cooperación para UNESCO en América Latina y el Caribe [Quality education in the digital age. An opportunity for cooperation for UNESCO in Latin America and the Caribbean]. https://n9.cl/kggxd

García-Contador, Y. \& Gutiérrez-Esteban, P. (2020). El rol docente en la sociedad digital [The teacher's role in the Digital Society]. Digital Education Review, 38(38), 1-22.

García-Sánchez, M, Reyes-Añorve, J, \& Godínez Alarcón, G. (2017). Las TIC en la educación superior, innovaciones y retos [ICT in higher education, innovations and challenges]. RICSH Revista Iberoamericana de las Ciencias Sociales y Humanísticas, 6(12).

Gonzalez, Z., Abad, M., \& Abad, E. (2020). El aula invertida: un desafío para la enseñanza universitaria [The flipped classroom: a challenge for university teaching]. Virtualidad Educacion Y Ciencia, 11(20), 75-91.

Martinez, D. (2018). ¿Enseñanza tradicional en el siglo XXI? [Traditional teaching in the 21st century?]. Revista Neuronum, 4(1), 1-8. 
Revista Arbitrada Interdisciplinaria KOINONIA

Año VI. Vol VI. N³. Edición Especial: Educación II. 2021

Hecho el depósito de Ley: FA2016000010 ISSN: 2542-3088

FUNDACIÓN KOINONIA (F.K). Santa Ana de Coro. Venezuela.

Matzumura-Kasano, J, Gutiérrez-Crespo, H, Pastor-García, C, Zamudio-Eslava, L, \& Ruiz-Arias, R. (2018). Metodología activa y estilos de aprendizaje en el proceso de enseñanza en el curso de metodología de la investigación de una facultad de ciencias de la salud [Active methodology and learning styles in the teaching process of the research methodology course of a faculty health sciences]. Anales de la Facultad de Medicina, 79(4), 293300. https://dx.doi.org/10.15381/anales.v79i4.15632

Munteanu, L. H., Gorghiu, G., \& Gorghiu, L. M. (2014). The Role of New Technologies for Enhancing Teaching and Learning in Arts Education. Procedia - Social and Behavioral Sciences, 122, 245-249. https://doi.org/10.1016/j.sbspro.2014.01.1336

Rivera, J. (2004). El aprendizaje significativo [Meaningful learning]. https://n9.cl/clk5

Rodríguez, J. (2013). Una mirada a la pedagogía tradicional y humanista [A look at traditional and humanist pedagogy]. https://n9.cl/jb4d

Rojas-Moreno, I. (2016). Formación y Profesionalización de la Docencia en el Nivel Superior en el Contexto de la Posmodernidad. Reflexiones sobre el Caso Mexicano [Training and Professionalization of Teaching at the Higher Level in the Context of Postmodernity. Reflections on the Mexican Case]. Revista Iberoamericana De Evaluación Educativa, 3(1e).

Serin, H. (2017). The Role of Passion in Learning and Teaching. International Journal of Social Sciences \& Educational Studies, 4(1), 60-64. https://doi.org/10.23918/ijsses.v4i1p60

Silva-da-Rocha-Marques, L. (2018). Active methodologies as strategies to develop education in values in nursing graduation. Escola Anna Nery, 22(3), 1-6. https://doi.org/10.1590/2177-9465-ean-2018-0023

Toledo, P., \& Sánchez, J. (2018). Aprendizaje Basado En Proyectos: Una Experiencia Universitaria [Project-Based Learning: A University Experience]. Profesorado, Revista de Currículum y Formación Del Profesorado, 22(2). https://doi.org/10.30827/profesorado.v22i2.7733

UNESCO. (2013). Enfoque Estratégico Sobre Tics En Educación En América Latina Y EI Caribe [Strategic Approach to ICTs in Education in Latin America and the Caribbean]. https://n9.cl/zt9r2 
Revista Arbitrada Interdisciplinaria KOINONIA

Año VI. Vol VI. N³. Edición Especial: Educación II. 2021

Hecho el depósito de Ley: FA2016000010 ISSN: 2542-3088

FUNDACIÓN KOINONIA (F.K). Santa Ana de Coro. Venezuela.

Wendy Elizabeth Rivadeneira-Ochoa; Luis Bolívar Cabrera-Berrezueta

UNESCO-OREALC. (2017). E2030: Educación y Habilidades para el Siglo XXI [E2030: Education and Skills for Siglo XXI]. https://n9.cl/hkrg

Zayas-Barreras, I, Parra Acosta, D, López Arciniega, R, \& Torres Sánchez, J. (2015). La innovación, competitividad y desarrollo tecnológico en las MIP y ME's del municipio de Angostura, Sinaloa [Innovation, competitiveness and technological development in SME's from the municipality of Angostura, Sinaloa]. Revista mexicana de ciencias agrícolas, 6(3), 603-617.

(C2021 por los autores. Este artículo es de acceso abierto y distribuido según los términos y condiciones de la licencia Creative Commons Atribución-NoComercial-Compartirlgual 4.0 Internacional (CC BY-NC-SA 4.0)

(https://creativecommons.org/licenses/by-nc-sa/4.0/). 